\title{
Effect of predicted low suspend pump treatment on improving glycaemic control and quality of sleep in children with type 1 diabetes and their caregivers: the QUEST randomized crossover study
}

Ulrike Schierloh ${ }^{1 *}$ (D, Gloria A. Aguayo ${ }^{2}$, Muriel Fichelle ${ }^{1}$, Cindy De Melo Dias ${ }^{1}$, Aljosa Celebic ${ }^{2}$, Michel Vaillant ${ }^{2}$, Katharine Barnard ${ }^{3}$, Ohad Cohen ${ }^{4}$ and Carine de Beaufort ${ }^{1}$

\begin{abstract}
Background: In attempting to achieve optimal metabolic control, the day-to-day management is challenging for a child with type 1 diabetes (T1D) and his family and can have a major negative impact on their quality of life. Augmenting an insulin pump with glucose sensor information leads to improved outcomes: decreased haemoglobin A1c levels, increased time in glucose target and less hypoglycaemia. Fear of nocturnal hypoglycaemia remains pervasive amongst parents, leading to chronic sleep interruption and lack of sleep for the parents and their children.

The QUEST study, an open-label, single-centre randomized crossover study, aims to evaluate the impact on time in target, in hypoglycaemia and hyperglycaemia and the effect on sleep and quality of life in children with T1D, comparing a sensor-augmented pump (SAP) with predictive low glucose suspend and alerts to the use of the same insulin pump with a flash glucose measurement (FGM) device not interacting with the pump.
\end{abstract}

Methods/design: Subjects meeting the inclusion criteria are randomized to treatment with the SAP or treatment with an insulin pump and independent FGM for 5 weeks. Following a 3-week washout period, the subjects cross over to the other study arm for 5 weeks. During the week before and in the last week of treatment, the subjects and one of their caregivers wear a sleep monitor in order to obtain sleep data. The primary endpoint is the between-arm difference in percentage of time in glucose target during the final 6 days of each treatment arm, measured by a blinded continuous glucose measurement (CGM).

Additional endpoints include comparison of quantity and quality of sleep as well as quality of life perception of the subjects and one of their caregivers in the two different treatment arms.

Recruitment started in February 2017. A total of 36 patients are planned to be randomized. The study recruitment was completed in April 2018.

Discussion: With this study we will provide more information on whether insulin pump treatment combined with more technology (SmartGuard ${ }^{\oplus}$ feature and alerts) leads to better metabolic control. The inclusion of indicators on quality of sleep with less sleep interruption, less lack of sleep and perception of quality of life in both children and their primary caregivers is essential for this study and might help to guide us to further treatment improvement.

\footnotetext{
* Correspondence: schierloh.ulrike@chl.lu

${ }^{1}$ Department of Pediatric Diabetes and Endocrinology, Clinique Pédiatrique,

Centre Hospitalier, Luxembourg City, Luxembourg

Full list of author information is available at the end of the article
}

(c) The Author(s). 2018 Open Access This article is distributed under the terms of the Creative Commons Attribution 4.0 International License (http://creativecommons.org/licenses/by/4.0/), which permits unrestricted use, distribution, and reproduction in any medium, provided you give appropriate credit to the original author(s) and the source, provide a link to the Creative Commons license, and indicate if changes were made. The Creative Commons Public Domain Dedication waiver (http://creativecommons.org/publicdomain/zero/1.0/) applies to the data made available in this article, unless otherwise stated. 
(Continued from previous page)

Trial registration: ClinicalTrials.gov, NCT03103867. Registered on 6 April 2017.

Keywords: Type 1 diabetes, Sleep, Quality of life, Children, Caregiver, Sensor-augmented insulin pump, Flash glucose measurement

\section{Type 1 diabetes Background}

Patients with type 1 diabetes (T1D) need lifelong insulin treatment and optimal metabolic control, which is essential to prevent short- and long-term complications [1]. To achieve optimal metabolic control, the day-to-day management is challenging for the children and their families and can have a major negative impact on their quality of life [2, 3].

Augmenting an insulin pump with glucose sensor information leads to improved outcomes. Whilst continuous interstitial glucose monitoring is associated with decreased haemoglobin A1c (HbA1c) levels and reduced time spent in hypoglycaemia in individuals with T1D using insulin pump therapy, better outcomes are associated with longer and continued use of the sensor [4]. Alerts are programmed and used in sensor-augmented pumps (SAPs) in order to inform patients and their caregivers about hypoglycaemic and hyperglycaemic events so that they can react quickly to such glycaemic excursions. However, alerts may be perceived as disturbing and may lead to diabetes distress and alert fatigue as well as continuous nocturnal awakenings [5].

Fear of nocturnal hypoglycaemia is common amongst parents of children with T1D, and it is associated with heightened vigilance by parents to regularly control their children's blood sugar values or to check the sensor information during the night $[6,7]$. This leads to chronic sleep interruption and to lack of sleep for the parents as well as for their children with diabetes [8]. Recent data show that 99\% of parents of children with T1D perform blood glucose checks on their child during the night to ensure their safety whilst sleeping [9]. This highly prevalent chronic sleep interruption affects both adults with T1D and parents/ carers of children with T1D, with resulting negative effects on their daily functioning and well-being [10]. Anxiety and fear of hypoglycaemia may have an impact on diabetes management and may complicate meeting glucose targets in patients with T1D [11, 12]. A recently published multicentre evaluation shows that SmartGuard ${ }^{\circ}$ technology significantly reduced the risk for hypoglycaemia in paediatric diabetes patients without increasing HbA1c [13].

The MiniMed $640 \mathrm{G}^{\circ}$ pump combines alerts with an automated insulin suspension; the pump suspends insulin infusion when the sensor glucose (SG) is within $3.9 \mathrm{mmol} / \mathrm{l}$ (70 $\mathrm{mg} / \mathrm{dl}$ ) above the low limit and predicted to be $1.1 \mathrm{mmol} / \mathrm{l}(20 \mathrm{mg} / \mathrm{dl})$ or lower above the low limit in $30 \mathrm{~min}$. Suspension lasts for a minimum of $30 \mathrm{~min}$ and until a 2-h suspension time is reached or the patient manually resumes basal rate infusion or auto-resumption occurs when the trend in glucose shifts and the SG is predicted to be $2.2 \mathrm{mmol} / \mathrm{l}(40 \mathrm{mg} / \mathrm{dl})$ above the threshold in $30 \mathrm{~min}$. Alerts can be set on or off; the low threshold alert is mandatory (SmartGuard ${ }^{\circ}$ ).

FreeStyle Libre ${ }^{\circ}$ is another device that continuously measures the interstitial glucose levels. The results can only be obtained when the patient/caregiver actively scans the sensor (flash glucose measurement, FGM). No alerts are given when glucose values increase or decrease, nor will information be available when the sensor is not scanned, and data are lost when more than $8 \mathrm{~h}$ elapse between scans. No communication exists between this glucose measurement and the insulin pump. The advantage of the FGM is intermittent access to 24-h glucose profiles without disturbing alerts.

Without any alerts, however, the symptoms of high or low glucose levels may be missed, and intervention delayed.

The impact of these technologies on metabolic control has been studied before [14]. We are not aware of any study evaluating their impact on quality of sleep in children and their caregivers, using questionnaire and Actigraph ${ }^{\circ}$ data.

\section{Objective}

The objective of this study is to evaluate whether the SAP (MiniMed $640 \mathrm{G}^{\circ}$ ) with SmartGuard ${ }^{\circ}$ feature increases time in glucose target and improves sleep quality and quantity and quality of life perception in patients with T1D and their primary caregivers, when compared with pump treatment with only continuous monitoring, FreeStyle Libre.

\section{Methods}

\section{Study design}

In this open-label, single-centre, randomized two-period crossover study, based in the Children's Hospital in Luxembourg, subjects with type 1 diabetes (6-14 years old, diabetes duration more than 6 months, on insulin pump for at least 6 months, HbA1c $\leq 11 \%$ ) are randomized to treatment with SAP with the SmartGuard $^{\circ}$ feature (MiniMed $^{\circ}$ 640G) or treatment with insulin pump and independent interstitial glucose measurement (FreeStyle Libre $^{\circ}$ ) for 5 weeks. Following a 3-week washout period, the subjects cross over to the other study arm for 5 weeks. 
The subjects and one of their caregivers will wear a sleep monitor $\left(\right.$ Actigraph $^{\odot}$ ) and complete a sleep diary the week before and during the last week of each treatment. This allows a comparison of the quality of sleep of the participants as well as the parents between the two treatment arms.

Randomization is performed by blocks of 8 with sequences $\mathrm{A}-\mathrm{B}$ and $\mathrm{B}-\mathrm{A}$ for the first and second periods respectively. Allocation is based on envelopes where the sequence code is concealed in advance. The order of the envelopes is determined by the randomization order and will allow allocation of the patient to one of the two sequences.

Reporting standards will be ensured by a rigorous quality process. The statistical process will include a statistical analysis plan approved by all members of the project team which will be applied for the statistical analysis of the study. Programming of the statistical analysis will be achieved and validated with a preliminary set of data and validated again when all data are available. Reporting will be done by the project statistician in a statistical report and validated by a second statistician.

The primary endpoint is the between-arm difference in percentage of time in glucose target during the final 6 days of each treatment arm, measured by a blinded continuous glucose measurement (CGM) (IPro $2^{\circledR}$ ).

Secondary endpoints include sleep duration, number of awakenings during the night and information about state of fatigue and activities in the daytime.

\section{Baseline and repeated assessment measures}

Demographic variables are sex, age, income, education and socio-economic status; anthropometric variables are weight $(\mathrm{kg})$ and height $(\mathrm{m})$. Quality of life perception will be assessed together with quality of sleep perception by questionnaires evaluating income, current professional activity, highest educational degree, hypoglycaemia fear, sleepiness and potential family responsibility [15-19].

\section{Procedures}

After they have received general information on the study aim and design, and also on the devices (pump, SmartGuard ${ }^{\oplus}$, FreeStyle Libre ${ }^{\oplus}$ and Actigraph ${ }^{\oplus}$ ), the parents and children who wish to participate in the study will be invited for the first visit (V0). The study timeline is shown in Fig. 1. The Standard Protocol Items: Recommendations for Interventional Trials (SPIRIT) schedule of interventions and assessments is shown in Fig. 2. The consent form (Additional file 1), trial questionnaires (Additional files 2, 3, 4 and 5), sleep diaries (Additional files 6, 7 and 8) and trial information (Additional files 9, 10, 11, 12, 13 and 14), as well as the populated SPIRIT checklist (Additional file 15).

At visit VO, after signing the informed consent/assent, the patients will be randomized to one of the two sequences (starting either with 640G with SmartGuard ${ }^{\circledR}$ or with FGM). Patients and one parent will be invited to fill out the questionnaires. A baseline $\mathrm{HbA1c}$ value and demographic and clinical data will be obtained in a case report form (CRF). During this period, all patients will be asked to perform a minimum of four capillary glucose

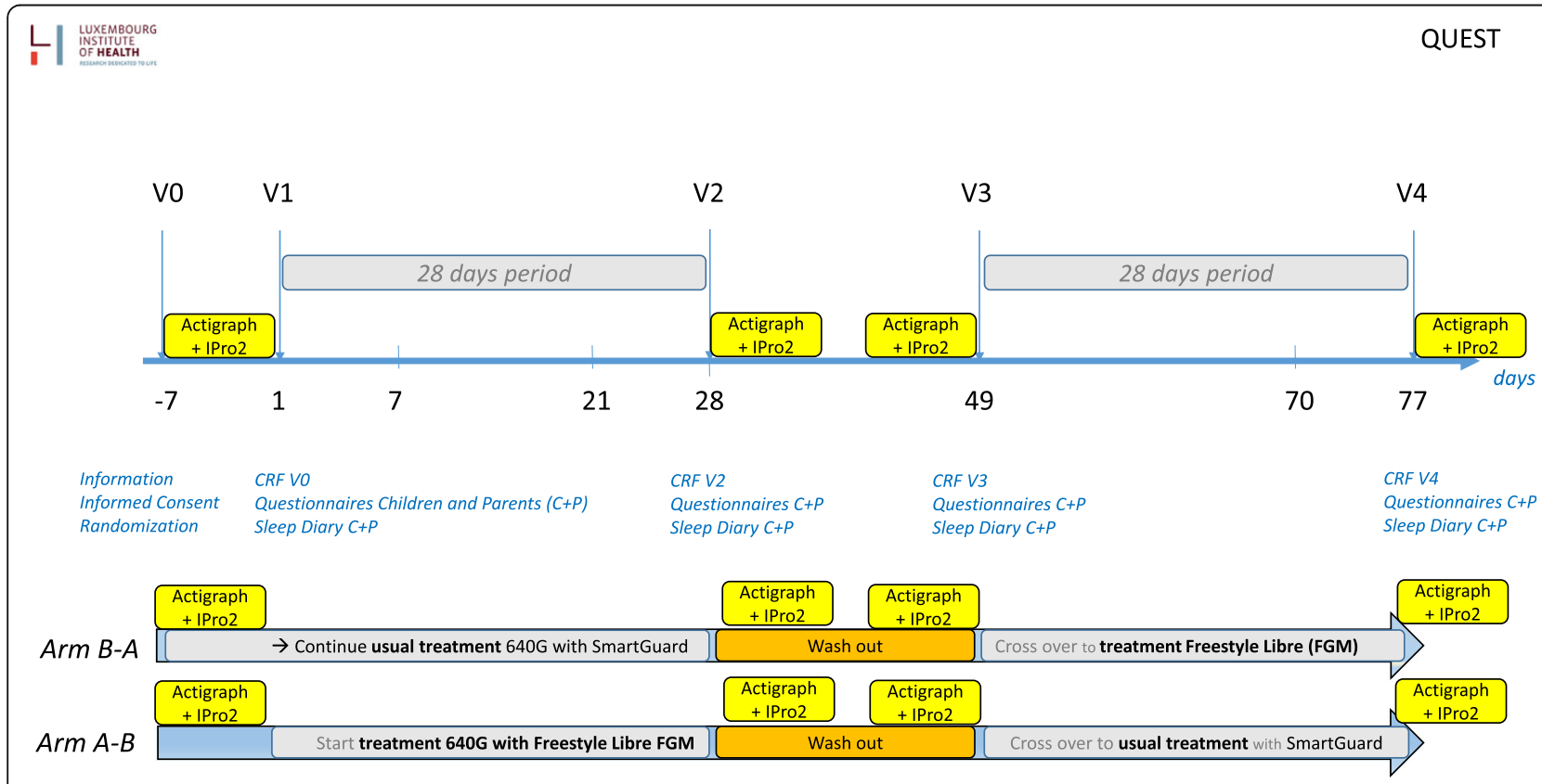

Fig. 1 Timeline of QUEST study 


\begin{tabular}{|c|c|c|c|c|c|c|c|}
\hline & Enrolment & Allocation & \multicolumn{5}{|c|}{ Post-allocation } \\
\hline TIMEPOINT & vo & vo & V1 & V2 & $\begin{array}{l}\text { Wash- } \\
\text { out }\end{array}$ & V3 & V4 \\
\hline \multicolumn{8}{|l|}{ ENROLMENT: } \\
\hline \multirow{2}{*}{$\begin{array}{l}\text { Eligibility screen } \\
\text { Informed consent }\end{array}$} & $X$ & & & & & & \\
\hline & $\mathrm{X}$ & & & & & & \\
\hline Information & $X$ & & & & & & \\
\hline Allocation & & $X$ & & & & & \\
\hline \multicolumn{8}{|l|}{ INTERVENTIONS: } \\
\hline Treatment $A-B$ & & & A & A & & B & B \\
\hline Treatment $B-A$ & & & B & B & & A & A \\
\hline \multicolumn{8}{|l|}{ ASSESSMENTS: } \\
\hline \multirow{2}{*}{ Actigraph/IPRO2 } & & $\mathrm{X}$ & & $X$ & & $X$ & $x$ \\
\hline & & $x$ & & $\mathrm{X}$ & & $\mathrm{X}$ & $x$ \\
\hline $\begin{array}{r}\text { Questionnaires/ } \\
\text { SleepDiaries }\end{array}$ & & $X$ & & $X$ & & $\mathrm{X}$ & $\mathrm{X}$ \\
\hline
\end{tabular}

Fig. 2 SPIRIT schedule of interventions and assessments

measurements daily (automatically registered in their pumps). No sensor (CGM or FGM) will be used during this week. The patient and one caregiver will be provided with an Actigraph ${ }^{\circ}$ and will be asked to fill out the sleep diaries during the 7 days of wearing the Actigraph ${ }^{\circ}$.

At the next visit (V1), the Actigraphs ${ }^{\bullet}$ will be collected for analysis. All patients will be started on the MiniMed Medtronic 640G pump, and either the SmartGuard ${ }^{\circ}$ or the FGM will be initiated.

As all patients are pump wearers, the transition towards the Medtronic 640G pump is not complicated. The use of the two glucose measurement tools will be discussed during the dedicated training session. A 24/7 diabetes hotline will be accessible for technical or any other issues.

Settings of the SmartGuard ${ }^{\circ}$ are standardized based on current experience [13]. The low limit will be set at $3.4 \mathrm{mmol} / \mathrm{l}(61 \mathrm{mg} / \mathrm{dl})$ with an insulin suspension at $\leq 7.3 \mathrm{mmol} / \mathrm{l}(131 \mathrm{mg} / \mathrm{dl})$ if the predicted value within $30 \mathrm{~min}$ is $4.5 \mathrm{mmol} / \mathrm{l}(81 \mathrm{mg} / \mathrm{dl})$. An alert before low will be set on to inform the parent/patient that insulin administration is suspended. It may take time for parents to develop confidence in the new technology; therefore, in this study, we decided to include the alert before low.

At the next visit, $V 2$ (after 4 weeks of treatment), the patients and families will be invited to complete the questionnaires. HbA1c values will be measured, and the CRF will be completed. The IPro2 ${ }^{\circ}$ for blinded CGM will be placed for 7 days, and the patient will be instructed to perform two glucose measurements/day for calibration. The Actigraphs ${ }^{\circ}$ will be provided. Patients and parents will be asked to fill out the sleep diaries during the following week.

This week will be followed by a washout period of 3 weeks.

During this period, the 640G pump will be maintained, but in combination with a minimum of four blood glucose measurements and no CGM or FGM.

Sleep assessment will be conducted with Actigraphs ${ }^{\circ}$ and sleep diaries 1 week before the start of the second treatment arm.

At visit V3, the second treatment period will be started on either FGM or SmartGuard ${ }^{\circ}$.

At visit V4, after 4 weeks of the treatment arm, the CRF and the questionnaires will be completed, the IPro2 ${ }^{\circ}$ for 
blinded CGM will be placed, and again the patient will be asked to perform two blood glucose measurements per day. Actigraphs $s^{\bullet}$ are provided, and patients and parents will be asked to fill out the sleep diaries.

After this week the devices will be collected for analysis, and the patient will restart his/her pre-study treatment.

Ennov Clinical software will be used for data management throughout the study. For the sleep analysis, Actilife ${ }^{\circ}$ software will be used.

\section{Data management and data quality}

Time in glucose target will be evaluated by the blinded CGM at the end of both treatment arms.

Data will be extracted from the blinded CGM with the Medtronic GlyVaRT software tool. The pump is uploaded to transfer information to Medtronic CareLink therapy management software through the use of a Contour Next Link $^{\ominus}$ glucose meter, which is also the uploading device.

Data quality will be ensured in the data management process. Double data entry will be performed in specific forms within Ennov Clinical, including online logical controls. A confrontation of both databases will be regularly carried out.

\section{Statistical analysis}

The percent time below glucose target, $<3.0 \mathrm{mmol} / \mathrm{l}$ $(54 \mathrm{mg} / \mathrm{dl})$ and $<2.5 \mathrm{mmol} / \mathrm{l}(45 \mathrm{mg} / \mathrm{dl})$, in glucose target (3.9-8 mmol/l, 70.2-144 $\mathrm{mg} / \mathrm{dl})$ and above glucose target ( $>10 \mathrm{mmol} / \mathrm{l}, 180 \mathrm{mg} / \mathrm{dl}$ ) during the final 6 days of a 5 week period will be compared between arms by using a linear model with treatment, sequence of treatments and period as fixed effects.

Sleep patterns will be assessed after sleep data validation by examining each sleep pattern. First, we will validate if the device was used a minimum of 5 days; second, we will analyse wear time and finally we will correct the sleep onset and morning wake-up according to data collected by the sleep diary.

Total sleep and wake time and number of awakenings at baseline, week 5 and week 13, in patients and at least one of their caregivers, will be analysed by using a linear mixed model with treatment given and period of treatment as fixed effects factors and patient as a random effect. The impact of family responsibility scale will be tested in the model, as well as time in target, age, gender and socioeconomic status and daily physical activity.

Quality of life perception and quality of sleep (Epworth Sleepiness Scale and sleep diary) in patients and in at least one of their caregivers in the two treatment arms at baseline, week 5 and week 13 will be analysed by using a linear model or a model for categorical outcome depending on the studied outcome.

The Hypoglycaemia Index for children and the Hypoglycaemia Fear Survey for parents/caregivers at baseline, week 5 and week 13 will also be analysed with the model specific to crossover trials.

A comparison of sleep diary data versus Actigraph ${ }^{\circ}$ data will be carried out.

Severe hypoglycaemia, defined by the International Society for Pediatric and Adolescent Diabetes (ISPAD) [12] will be analysed through a table of frequencies.

Total sleep time will be analysed using a linear mixed model with treatment given and period of treatment as fixed effects factor and patient as a random effect. Sleep analysis will be performed with Actilife ${ }^{\circ}$ Software calculating duration of sleep during day and night and number of awakenings, in comparison with the sleep diaries.

\section{Sample size}

Based on paediatric data, the percent time spent in glucose target $(3.9-8 \mathrm{mmol} / \mathrm{l})$ in the paediatric population is estimated to be $40-50 \%$. Assuming that an increase of $10-15 \%$ in time in glucose target is considered as clinically meaningful, a significance level set at 5\% (two sided) and a power of $80 \%$, a minimum number of patients of 31 per group would be necessary. Taking into account the within-subject standard deviation and a maximum 10\% of dropouts, a sample size of 36 patients should be included in the study.

Timeline/recruitment/checklist

Ethical approval for the final study was obtained in January 2017. Recruitment started in February 2017. The study was completed in April 2018. The timeline of the study is shown in Fig. 1. A SPIRIT checklist for this study protocol is included as Additional file 15.

\section{Discussion}

With this study we will provide more information on whether insulin pump treatment combined with more technology (SmartGuard ${ }^{\circ}$ feature and alerts) leads to better metabolic control. As diabetes is a chronic disease and will require lifelong treatment, the impact of quality of life and sleep may play a role in treatment adherence and outcome. The inclusion of indicators on quality of sleep with less sleep interruption, less lack of sleep and perception of quality of life in both children and their primary caregivers is essential for this study and might help to guide us to further treatment improvement.

Trial status at time of manuscript re-submission in October 2018

Patient recruitment has been completed.

\section{Additional files}

Additional file 1: Patient Information and consent form. (DOCX $233 \mathrm{~kb}$ ) Additional file 2: Questionnaires for parents. (DOC $158 \mathrm{~kb}$ ) 


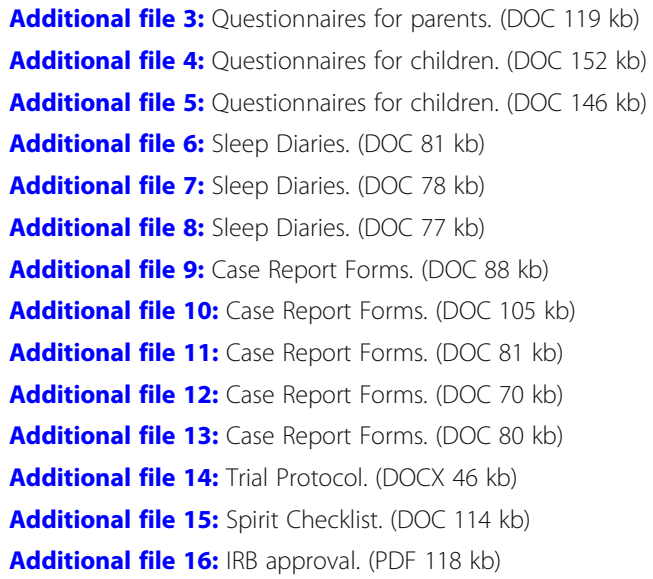

\section{Abbreviations}

CGM: Continuous glucose measurement; CRF: Case report form; FGM: Flash glucose measurement; HbA1c: Haemoglobin A1c; ISPAD: International Society for Pediatric and Adolescent Diabetes; SAP: Sensor-augmented pump; SG: Sensor glucose

\section{Acknowledgements}

Not applicable.

\section{Funding}

This is an investigator initiated study. Medtronic contributed in kind to the devices (insulin pumps, transmitter, IPro2) and to the accessories (glucose sensors).

\section{Availability of data and materials}

The datasets generated and analysed during the current study are available from the corresponding author on reasonable request.

\section{Authors' contributions}

US and $\mathrm{CdB}$ conceived the study and design and the protocol, and performed recruitment, data analysis and writing of the paper. MF and CDMD performed recruitment and conduct of the study. GAA, AC and MV performed study design, data management and data analysis. KB and OC worked on the protocol. All authors read and approved the final manuscript.

\section{Ethics approval and consent to participate}

A statement on ethics approval and consent is attached (Additional file 16). The name of the Luxembourg Ethics committee is CNER (Comité national d'Ethique de Recherche)

\section{Consent for publication}

Consent forms were obtained from each participant and his participating parent.

\section{Competing interests}

The authors declare that they have no competing interests.

\section{Publisher's Note}

Springer Nature remains neutral with regard to jurisdictional claims in published maps and institutional affiliations.

\section{Author details}

'Department of Pediatric Diabetes and Endocrinology, Clinique Pédiatrique, Centre Hospitalier, Luxembourg City, Luxembourg. ${ }^{2}$ Luxembourg Institute of Health, Luxembourg City, Luxembourg. ${ }^{3}$ Bournemouth University, Bournemouth, UK. ${ }^{4}$ Medtronic Diabetes, Tolochenaz, Switzerland.
Received: 5 April 2018 Accepted: 1 November 2018 Published online: 04 December 2018

\section{References}

1. Diabetes Control and Complications Trial Research Group. The effect of intensive treatment of diabetes on the development and progression of long-term complications in insulin-dependent diabetes mellitus. N Engl J Med. 1993:329:977-86.

2. Wood JR, Miller KM, Maahs DM, Beck RW, DiMeglio LA, Libman IM, Quinn M, Tamborlane W, Woerner SE, T1D Exchange Clinic Network. Most youth with type 1 diabetes in the T1 D Exchange Clinic Registry do not meet American Diabetes Association or International Society for Pediatric and Adolescent Diabetes clinical guidelines. Diabetes Care. 2013;36(7):2035-7.

3. Whittemore R, Jaser S, Chao A, Jang M, Grey M. Psychological experience of parents of children with type 1 diabetes: a systematic mixed-studies review. Diabetes Educ. 2012;38(4):562-79.

4. Battelino T, Conget I, Olsen B, Schütz-Fuhrmann I, Hommel E, Hoogma R, Schierloh U, Sulli N, Bolinder J, SWITCH Study Group. The use and efficacy of continuous glucose monitoring in type 1 diabetes treated with insulin pump therapy: a randomized controlled trial. Diabetologia. 2012;55(12):3155-62.

5. Lindström C, Åman J, Anderzén-Carlsson A, Lindahl Norberg A. Group intervention for burnout in parents of chronically ill children - a smallscale study. Scand J Caring Sci. 2016;30(4):678-86.

6. Barnard K, Thomas S, Royle P, Noyes K, Waugh N. Fear of hypoglycaemia in parents of young children with type 1 diabetes: a systematic review. BMC Pediatr. 2010:10(50):50-7.

7. Barnard KD, Oliver N, Adolfsson P, Aldred C, Kerr D. Is iatrogenic sleep disturbance worth the effort in type 1 diabetes? Diabet Med. 2015;32(8):984-6.

8. Lindström C, Aman J, Norberg AL. Parental burnout in relation to sociodemographic, psychosocial and personality factors as well as disease duration and glycaemic control in children with type 1 diabetes mellitus. Acta Paediatr. 2011;100(7):1011-7.

9. Barnard K, James J, Kerr D, Adolfsson P, Runion A, Sebedzija G. Impact of chronic sleep disturbance for people living with T1 diabetes. J Diabetes Sci Technol. 2016;10(3):762-7.

10. Jaser SS, Foster NC, Nelson BA, Kittelrud JM, DiMeglio LA, Quinn M, Willi SM, Simmons JH, T1D Exchange Clinic Network. Sleep in children with type 1 diabetes and their parents in the T1D Exchange. Sleep Med. 2017;39:108-15.

11. Wild D, von Maltzahn R, Brohan E, Christensen T, Clauson P, GonderFrederick $L$. A critical review of the literature on fear of hypoglycemia in diabetes: implications for diabetes management and patient education. Patient Educ Couns. 2007:68(1):10-5.

12. Ly TT, Maahs DM, Rewers A, Dunger D, Oduwole A, Jones TW, International Society for Pediatric and Adolescent Diabetes. ISPAD Clinical Practice Consensus Guidelines 2013. Assessment and management of hypoglycemia in children and adolescents with diabetes. Pediatr Diabetes. 2014;15(Suppl 20):180-92.

13. Biester T, Kordonouri $\mathrm{O}$, Holder M, Remus K, Kieninger-Baum D, Wadien T, Danne T. "Let the algorithm do the work": reduction of hypoglycemia using sensor-augmented pump therapy with predictive insulin suspension (SmartGuard) in pediatric type 1 diabetes patients. Diabetes Technol Ther. 2017;19(3):173-82

14. Abraham MB, de Bock M, Paramalingam N, O'Grady MJ, Ly TT, George C, Roy A, Spital G, Karula S, Heels K, Gebert R, Fairchild JM, King BR, Ambler GR, Cameron F, Davis EA, Jones TW. Prevention of insulin-induced hypoglycemia in type 1 diabetes with predictive low glucose management system. Diabetes Technol Ther. 2016;18(7):436-43.

15. Kamps $\lrcorner$, Roberts MC, Varela RE. Development of a new fear of hypoglycemia scale: preliminary results. J Pediatr Psychol. 2005;30(3):287-91.

16. Haugstvedt A, Wentzel-Larsen T, Aarflot M, Rokne B, Graue M. Assessing fear of hypoglycemia in a population-based study among parents of children with type 1 diabetes - psychometric properties of the hypoglycemia fear survey - parent version. BMC Endocr Disord. 2015;15:2.

17. Patton SR, Noser AE, Clements MA, Dolan LM, Powers SW. Reexamining the hypoglycemia fear survey for parents of young children in a sample of children using insulin pumps. Diabetes Technol Ther. 2017;19(2):103-8.

18. Johns MW. A new method for measuring daytime sleepiness: The Epworth Sleepiness Scale. Sleep. 1991;14(6):540-5.

19. Cameron FJ, Skinner TC, De Beaufort CE, Hoey H, Swift PG, Aanstoot H, Aman J, Martul P, Chiarelli F, et al. Hvidoere Study Group on Childhood Diabetes. Are family factors universally related to metabolic outcomes in adolescents with type 1 diabetes? Diabet Med. 2008;25(4):463-8. 